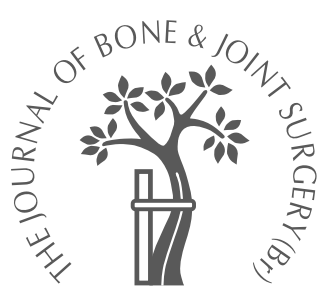

E. Calvo, J. J. Granizo, D. FernándezYruegas

From the Fundación Jiménez Diaz, Madrid, Spain
E. Calvo, MD, PhD, Orthopaedic Surgeon D. Fernández-Yruegas, MD, Orthopaedic Surgeon Department of Orthopaedic Surgery

J. J. Granizo, MD

Epidemiologist

Department of Epidemiology Fundación Jiménez Diaz, Avenue Reyes Católicos 2, 28040 Madrid, Spain.

Correspondence should be sent to Dr E. Calvo; e-mail: ecalvo@fjd.es

(C)2005 British Editorial

Society of Bone and

Joint Surgery

doi:10.1302/0301-620X.87B5.

$15794 \$ 2.00$

$J$ Bone Joint Surg [Br] 2005;87-B:677-83.

Received 5 July 2004;

Accepted after revision

25 August 2004

\title{
Criteria for arthroscopic treatment of anterior instability of the shoulder
}

\author{
A PROSPECTIVE STUDY
}

We prospectively evaluated 61 patients treated arthroscopically for anterior instability of the shoulder at a mean follow-up of 44.5 months (24 to 100) using the Rowe scale. Those with post-operative dislocation or subluxation were considered to be failures. Logistic regression analysis was used to identify patients at increased risk of recurrence in order to develop a suitable selection system.

The mean Rowe score improved from 45 pre-operatively to 86 at follow-up $(p<0.001)$. At least one episode of post-operative instability occurred in 11 patients (18\%), although their stability improved ( $p=0.018$ ), and only three required revision. Subjectively, eight patients were dissatisfied. Age younger than 28 years, ligamentous laxity, the presence of a fracture of the glenoid rim involving more than $15 \%$ of the articular surface, and post-operative participation in contact or overhead sports were associated with a higher risk of recurrence, and scored 1, 1, 5 and 1 point, respectively. Those patients with a total score of two or more points had a relative risk of recurrence of $43 \%$ and should be treated by open surgery.

Arthroscopic Bankart repair for the treatment of instability of the shoulder has become increasingly popular as it is less invasive than open surgery and produces a better cosmetic result, range of movement and function. ${ }^{1,2}$ Although it achieved excellent early results, comparative studies have subsequently reported higher rates of failure than those of open surgery. ${ }^{2-4}$ Guidelines for selection of suitable candidates for an arthroscopic stabilisation have been suggested. ${ }^{4-13}$ Many studies are retrospective and lack strict selection criteria and consistency or have a short follow-up. Consequently, the indications for arthroscopic Bankart stabilisation are not well defined. In spite of these limitations, the technique appears best for patients with anterior unidirectional instability. ${ }^{3,12}$ Instability of the shoulder usually affects young, active patients. Consequently, it is important to evaluate the function and level of satisfaction obtained with open techniques which have a lower failure rate, but carry a risk of restriction of movement and a longer period of recovery.

Our aim was to develop a selection system for arthroscopic Bankart repair based upon an analysis of the factors which may determine the result, including the level of satisfaction and the degree of stability achieved.

\section{Patients and Methods}

A total of 65 patients with anterior instability of the shoulder underwent a Bankart repair using an arthroscopic transglenoid multiplesuture technique between April 1995 and December 2001.

Before inclusion, the patients were informed about the advantages and disadvantages of arthroscopic stabilisation in comparison with those of open surgery. All gave informed consent, and an institutional review board approved the study. One patient chose to undergo open repair and three patients treated arthroscopically were lost to follow-up. This left a total of 61 patients (52 men and nine women) for review. No patient received bilateral surgery in this study, although four had symptoms of contralateral instability, one of whom had received earlier surgery. The mean age at the time of operation was 27.5 years (17 to 67 ) and the mean follow-up was 44.5 months (24 to 100$)$.

Inclusion and exclusion criteria. The criteria for inclusion in the study were as follows. First, presence of unidirectional anterior instability of the shoulder which interfered with daily living or athletic activity. The diagnosis was made according to the criteria defined by Gartsmann, Roddey and Hammerman: ${ }^{14}$ 1) a description of dislocation or a feeling of loose- 
ness and slipping; 2) pain and/or apprehension when on testing for anterior instability; 3) radiological evidence of glenohumeral instability; and 4) findings during examination under anaesthesia or arthroscopic operation which indicated anterior glenohumeral instability. Secondly, each patient had to have suffered at least two dislocations of the shoulder. Thirdly, the minimum follow-up period had to be two years. Finally, the patients had to have undergone repair using a transglenoid multiple-suture technique. ${ }^{15}$

Patients were excluded if they had: 1) posterior, inferior, or multidirectional instability; 2) voluntary glenohumeral instability (this group was managed non-operatively); 3) previous failed arthroscopic surgery requiring an open procedure; 4) arthroscopically-documented lesions not addressed in our study but potentially linked to instability (superior labrum anterior to posterior, (SLAP) and humeral avulsion of the ligaments); 5) fractures involving more than $30 \%$ of the articular surface of the glenoid and which had undergone open reconstruction; and 6) conditions unrelated to instability, such as tears of the rotator cuff or damage to biceps involving more than $50 \%$ of the substance of its tendon.

Pre-operative assessment, surgical technique and postoperative management. The direction of instability was assessed pre-operatively. We noted the position of the arm which produced pain or apprehension. This, combined with the history, radiological findings, and pre-operative examination under anaesthesia, allowed categorisation of the direction of instability. Anterior translation was assessed by applying an anterior force to the shoulder with the arm in $90^{\circ}$ of abduction. Posterior translation was examined by applying a posterior force with the arm elevated to $90^{\circ}$, in $30^{\circ}$ of abduction and in approximately $90^{\circ}$ of internal rotation. Inferior translation was evaluated by applying an inferior force to the shoulder with the arm in $0^{\circ}$ of abduction (the sulcus test). Patients with excessive anterior, inferior, and posterior translation of the humeral head were considered to have multidirectional instability and scheduled for open capsular shift. Both shoulders were examined for instability. Anteroposterior radiography of the shoulder and MRI were carried out on all patients. Radiological evidence of anterior glenohumeral instability consisted of a finding of an anteriorly dislocated humeral head. Evidence of anterior instability on MRI included an avulsion of the anterior labrum from the glenoid (Bankart lesion), an anterior labroligamentous periosteal sleeve avulsion, a fracture of the anterior glenoid rim, or a Hill-Sachs lesion. Before surgery, all patients were examined under anaesthesia to determine the direction of instability and only those with anterior instability were included in the study.

Arthroscopy of the shoulder was performed under general anaesthesia with the patient in the lateral position. A complete glenohumeral arthroscopic examination preceded the stabilisation. The transglenoid suture technique was performed as a modification of Caspari's method. ${ }^{7,8,15}$ If the labrum or capsule was detached from the margin of the glenoid, the remaining labrum was debrided of fibrous tissue and the anterior capsule dissected until it could be reattached superolaterally to its anatomical insertion. If a bony Bankart lesion was present, the detached bone was resected with a motorised burr until the remaining labrum and capsule were free. Partial tears of the tendons of the cuff or the long head of biceps were debrided when present. If there was no labral detachment and the primary finding was capsular laxity, this was treated by capsulorraphy. The anterior capsule was divided just lateral to the labrum from approximately the 2 to 6 o'clock position in a right shoulder or the 6 to 10 o'clock position in a left shoulder, and a portion of capsule resected. The free edge of the capsule was then mobilised and repaired to the glenoid margin adjacent to the labrum. The examination under anaesthesia, detailed arthroscopic findings, and procedural details were documented on a standardised form.

The post-operative regime was similar for all patients. After surgery the patients kept the arm in an internal rotation sling for four weeks. Active exercises for fingers, wrist, and elbow, as well as isometric exercises of the deltoid muscle were started one day after surgery. At two weeks, the patients removed their sling for bathing, and passive forward elevation to $90^{\circ}$ and external rotation to neutral was started. Four weeks after operation, active and passive movements began and at eight weeks more vigorous exercises were started. A complete return to normal daily activities was allowed three months after surgery, with ordinary sporting activity being allowed at six months and contact or overhead sports at nine months. No post-operative imaging studies were routinely performed.

Clinical objective evaluation and recurrent instability. All patients were prospectively evaluated with a minimum follow-up of two years by an independent, blinded observer using the rating system of Rowe, Patel and Southmayd. ${ }^{16}$ This is a 100-point scale which assigns 50 points for function, 30 for stability, 10 for movement, and 10 for pain. A score of 90 to 100 points was deemed to be excellent, 75 to 89 good, 50 to 74 fair, and less than 50 poor. Because this scale assigns points for a range of movement based on a percentage of normal, the range of movement of the contralateral shoulder was used for the normal values. In the patient who had undergone surgery on the opposite shoulder, $90^{\circ}$ of external rotation, $50^{\circ}$ of internal rotation, and $180^{\circ}$ of abduction were considered to be normal values. Pre- and post-operative scores were compared using the Wilcoxon test.

Recurrence was defined as either subluxation or dislocation after surgery. A positive apprehension sign did not indicate failure if the shoulder was otherwise subjectively stable and shoulder function had been restored. The number of dislocations and subluxations, the time elapsed from surgery, as well as the mechanism of recurrence were also recorded in those patients with post-operative instability. Patient selection. Based upon previously reported findings, 12 variables which might determine the outcome of surgical 
treatment were studied. ${ }^{3-13,17-25}$ These were the age at surgery, the age at first dislocation, gender, dominance, aetiology of instability, number of dislocations and subluxations, ligamentous laxity, presence of a Bankart lesion, fracture of the anterior glenoid rim or a Hill-Sachs lesion, the number of sutures, and post-operative participation in contact or overhead sports.

The aetiology of instability was classified as either traumatic or atraumatic according to the criteria described by Wirth, Blatter and Rockwood. ${ }^{26}$ A traumatic aetiology was suggested when the injury had occurred with the arm forcefully abducted, externally rotated and extended and there had been acute pain, the need for manipulative reduction, and residual aching for several weeks. Atraumatic instability was characterised either by an insidious onset or the development of symptoms after minor trauma and was associated with mild pain and spontaneous reduction. The total number of episodes, and type of instability, were recorded. Subluxation was defined as a 'dead-arm' phenomenon or instability which spontaneously reduced. Those episodes which required reduction by health-care professionals were considered to be dislocations. Patients were classified as having ligamentous laxity if two of the following three findings were demonstrated on examination: 1) hyperextension of the elbow beyond neutral, 2) hyperextension of the index metacarpophalangeal joint beyond $90^{\circ}$, and 3) hyperabduction of the thumb to the forearm. ${ }^{27}$ The presence of a Bankart lesion, capsular laxity, fracture of the anterior glenoid rim and Hill-Sachs lesion were documented at arthroscopy. A Bankart lesion was considered to be present if there was a detachment of the antero-inferior labrum from its anatomical position on the glenoid rim. Isolated fraying of the labrum was not considered to be a Bankart lesion. The quality of the capsular tissue was assessed by direct inspection and palpation with a proble. The presence of a distinct anterior band of the inferior and middle glenohumeral ligaments was considered to reflect a competent capsule. Capsular laxity was indicated if a positive drive-through sign was present or the antero-inferior capsule was sufficiently patulous to produce a large pouch when the joint was distended. Fractures of the anterior glenoid rim were arthroscopically confirmed to involve less than $30 \%$ of the surface of the joint when present, and were classified as type 1 or type 2 if they involved less than $15 \%$ or $16 \%$ to $30 \%$ of the articular surface, respectively.

Statistical analysis. These variables were associated with recurrent post-operative instability in order to develop a reliable system for selection of the patients. Univariate analysis was first performed to calculate the association between the recurrence of post-operative instability and the independent variables analysed. Fisher's exact test was used for categorical variables and the Mann-Whitney test for quantitative variables. Logistic regression analysis was undertaken to determine the significant predictors for recurrence, and relations at $p$ values of less than 0.15 were retained for that purpose. Backward selection and stepwise approaches were used in a model to predict recurrence. Scores were assigned by rounding the odds ratio estimates to the nearest integer. Sensitivity and specificity were calculated for each breakpoint. To ensure that few subjects with a high risk of recurrence would be missed, exact binomial confidence intervals (CI) were calculated, and threshold scores were chosen to yield a sensitivity of $90 \%$ or greater. Analyses were performed using SPSS statistical software system version 11.5 (SPSS Inc, Chicago, Illinois).

Degree of instability, activity level and subjective evaluation. Pre- and post-operative levels of activity and instability were in order to evaluate the degree compared using the Wilcoxon test stabilisation achieved by surgery. The level of instability was graded according to the method described by Manta et al, ${ }^{6}$ which determines the least strenuous activity that produces symptoms. Level 1 indicates that a patient has occasional shoulder instability after a traumatic event or a forceful movement of the shoulder; level 2, instability with sports; level 3, instability with activities of daily living; and level 4, instability during sleep, or spontaneous instability. Regarding levels of activity, patients were grouped into one of the five categories: type 0 , a sedentary life without sporting activity; type 1 , non-contact sports, such as swimming the breaststroke, rowing, bowling or running; type 2, sports which do not require overhead use of the arm, such as riding a bicycle, motorcycling, or water skiing; type 3 , sports which require overhead use of the arm without forced abduction and external rotation, including swimming the crawl, weight-lifting, golf or sailing; type 4, sports which include overhead hitting movements, or contact sports with a high risk of falls such as basketball, handball, volleyball, tennis, soccer, downhill skiing, rugby, judo, and karate. The level of sports participation was categorised as recreational (level 1), high-school team sport (level 2), college team sport (level 3), or professional (level 4).

For the subjective evaluation, all patients were requested to score the result of their operation as excellent, good, fair or bad, and to indicate if they would have had the procedure again for the same problem. Those who rated the result of surgery as fair or bad were considered to be dissatisfied. Finally, because revision open surgery was offered to all patients who suffered further instability, those who decided not to be revised were asked to explain why.

\section{Results}

Clinical objective evaluation and recurrent instability. The mean Rowe ${ }^{16}$ score increased significantly from 45 points pre-operatively to 86 points after operation $(\mathrm{p}<0.001)$. The overall objective results were excellent in 42 (68.9\%), good in eight $(13.1 \%)$, fair in seven $(11.5 \%)$, and poor in four patients $(6.5 \%)$. Seven patients who were rated as good had no post-operative instability, but six showed mild discomfort in abduction and external rotation, and one had an external rotation deficit of $30^{\circ}$. The 11 patients $(18 \%)$ with poor or fair results had a recurrence of instability dur- 
Table I. Univariate analysis. Variables associated with outcome

\begin{tabular}{|c|c|c|c|}
\hline Variable & No recurrence & Recurrence & p value \\
\hline Mean age at surgery in yrs $( \pm \mathrm{SD})$ & $28.5 \pm 8.7$ & $22.6 \pm 4.3$ & 0.03 \\
\hline \multicolumn{4}{|l|}{ Age at surgery in yrs } \\
\hline$\geq 28$ & 23 & 1 & \multirow[t]{2}{*}{0.02} \\
\hline$\leq 27$ & 27 & 11 & \\
\hline Mean age at first dislocation in yrs $( \pm S D)$ & $21.6 \pm 8.2$ & $19.4 \pm 3.8$ & 0.38 \\
\hline \multicolumn{4}{|l|}{ Gender (number) } \\
\hline Male & 41 & 11 & \multirow[t]{2}{*}{0.19} \\
\hline Female & 9 & 0 & \\
\hline \multicolumn{4}{|l|}{ Dominant limb operated (number) } \\
\hline Yes & 39 & 8 & \multirow[t]{2}{*}{0.70} \\
\hline No & 11 & 3 & \\
\hline \multicolumn{4}{|l|}{ Aetiology of instability (number) } \\
\hline Traumatic & 46 & 10 & \multirow[t]{2}{*}{1.0} \\
\hline Atraumatic & 4 & 1 & \\
\hline \multicolumn{4}{|c|}{ Mean number of pre-operative episodes of instability $( \pm S D)$} \\
\hline Total & $11.3 \pm 9.1$ & $12.1 \pm 9.2$ & 0.80 \\
\hline Dislocations & $3.6 \pm 5.1$ & $5.5 \pm 3.5$ & 0.26 \\
\hline Subluxations & $7.7 \pm 9.2$ & $6.6 \pm 6.7$ & 0.72 \\
\hline \multicolumn{4}{|l|}{ Number of pre-operative dislocations } \\
\hline$\geq 6$ & 9 & 4 & \multirow[t]{2}{*}{0.08} \\
\hline$\leq 5$ & 41 & 7 & \\
\hline \multicolumn{4}{|l|}{ Ligamentous laxity (number) } \\
\hline Yes & 39 & 6 & \multirow[t]{2}{*}{0.11} \\
\hline No & 11 & 5 & \\
\hline \multicolumn{4}{|l|}{ Bankart lesion (number) } \\
\hline Yes & 43 & 11 & \multirow[t]{2}{*}{0.33} \\
\hline No & 7 & 0 & \\
\hline \multicolumn{4}{|l|}{ Fracture of the anterior glenoid rim (number) } \\
\hline Type 1 & 5 & 0 & \multirow{3}{*}{$0.01^{*}$} \\
\hline Type 2 & 0 & 2 & \\
\hline None & 45 & 9 & \\
\hline \multicolumn{4}{|l|}{ Hill-Sachs lesion (number) } \\
\hline Yes & 36 & 7 & \multirow[t]{2}{*}{0.71} \\
\hline No & 14 & 4 & \\
\hline Mean number of sutures $( \pm \mathrm{SD})$ & $4.5 \pm 1.5$ & $3.8 \pm 1.8$ & 0.15 \\
\hline \multicolumn{4}{|c|}{ Post-operative participation in contact or overhead sports (number) } \\
\hline Yes & 12 & 7 & \multirow[t]{2}{*}{0.03} \\
\hline No & 38 & 4 & \\
\hline
\end{tabular}

ing follow-up. Five patients had multiple post-operative episodes of instability, whereas the remaining six had a single episode. Recurrences occurred at a mean post-operative time of 26 months ( 6 to 46 ), and half appeared more than two years after surgery. Post-operative instability developed after a traumatic event in all these patients, in nine as a result of sporting activities or a fall, and in two during an epileptic seizure. Only three patients with multiple episodes of recurrent instability requested revision. One was found to have a lax capsule without a Bankart lesion and was treated by an open capsular shift, one had a Bankart lesion and was managed by an open Bankart repair, and one who had a fracture of the anterior glenoid rim underwent a Bristow operation.

Post-operatively, no patient lost more than $5^{\circ}$ of flexion, abduction or internal rotation. The mean loss of external rotation was $5.5^{\circ}$ (0 to 30 ). No major intra-operative or peri-operative complications occurred. Two patients had a transient neurapraxia of the axillary and musculocutaneous nerves, respectively, which resolved within a few weeks without consequences. One patient underwent manipula- tion under anaesthesia because of post-operative stiffness of the shoulder and a full range of movement was achieved. Twelve patients noted mild discomfort in the infraspinatus fossa because of subcutaneous protrusion of the knot, which required excision in two.

Patient selection system. The results of the univariate analysis of the variables related to poor outcome are given in Table I. Age less than 28 years, ligamentous laxity, more than five pre-operative dislocations, the presence of a fracture of the anterior glenoid rim involving more than $15 \%$ of the articular surface (type 2), and post-operative participation in contact or overhead sports were associated with failure ( $\mathrm{p}<0.15)$, and were considered for inclusion in the logistic regression analysis. In the stepwise logistic regression analysis age less than 28 years, the presence of ligamentous laxity, type- 2 fracture of the glenoid rim and participation post-operatively in high-risk sports predicted the recurrence of instability (Table II). The model goodness of fit was $r=0.334$. They scored $1,1,5$ and 1 point, respectively, and the discriminatory performance of a scoring system with these variables is presented in Table III. Using two 
Table II. Variables included in the stepwise logistic regression analysis (predictors of recurrence)

\begin{tabular}{|c|c|c|c|c|c|}
\hline Variable & Odds ratio & $95 \%$ confidence interval & p value & $\mathbf{R}$ & Score \\
\hline Age $<28$ yrs & 8.59 & (0.78 to 94.97$)$ & 0.08 & 0.14 & 1 \\
\hline Ligamentous laxity & 6.01 & (0.92 to 39.20$)$ & 0.06 & 0.16 & 1 \\
\hline Type-2 fracture of the anterior glenoid rim & 81.106 & $(1.07 \text { to } \mathrm{NC})^{*}$ & $0.93^{\dagger}$ & 0.01 & 5 \\
\hline Post-operative participation in contact or overhead sports & 12.07 & (1.71 to 85.24$)$ & 0.01 & 0.27 & 1 \\
\hline
\end{tabular}

* not calculable

$\dagger$ the statistical significance of this variable was poor because of the size of the sample

Table III. Discriminatory performance of recurrence risk assessment algorithms, by total score (percentage) and 95\% confidence interval

\begin{tabular}{lcccc}
\hline Score & Sensitivity (95\% Cl) & Specificity (95\% Cl) & PPV $^{*}$ (95\% Cl) & NPV $^{\dagger}$ (95\% Cl) \\
\hline$\geq 1$ & $100.0(100.0$ to 100.0$)$ & $36.0(22.7$ to 49.3$)$ & $43.5(12.5$ to 38.6$)$ & $100.0(100.0$ to 100.0$)$ \\
$\geq 2$ & $90.9(73.9$ to 100.0$)$ & $74.0(61.8$ to 86.1$)$ & $83.3(53.5$ to 100.7$)$ & $97.4(92.3$ to 100.0$)$ \\
$\geq 3$ & $45.5(16.0$ to 74.9$)$ & $98.0(94.1$ to 100.0$)$ & $100.0(100.0$ to 100.0$)$ & $89.1(80.6$ to 97.3$)$ \\
$\geq 6$ & $18.2(0.0$ to 41.0$)$ & $100.0(100.0$ to 100.0$)$ &
\end{tabular}

PPV positive predictive value

† NPV, negative predictive value

Table IV. Pre- and post-operative instability, sporting activity and competition levels, by number of patients

\begin{tabular}{|c|c|c|c|c|c|c|}
\hline \multirow[b]{2}{*}{ Level } & \multicolumn{2}{|l|}{ Instability level } & \multicolumn{2}{|c|}{ Sporting activity } & \multicolumn{2}{|c|}{ Competition level } \\
\hline & Pre-operative & Post-operative & Pre-operative & Post-operative & Pre-operative & Post-operative \\
\hline 0 & 0 & 50 & 1 & 13 & 1 & 13 \\
\hline 1 & 2 & 7 & 8 & 6 & 48 & 42 \\
\hline 2 & 16 & 1 & 4 & 7 & 2 & 2 \\
\hline 3 & 20 & 2 & 11 & 16 & 7 & 2 \\
\hline 4 & 23 & 1 & 37 & 19 & 3 & 2 \\
\hline
\end{tabular}

or more points as breakpoint the sensitivity was $90.9 \%$ and the specificity $74.0 \%$, with a probability of recurrence of $44 \%$. The presence of a type- 2 fracture of the glenoid showed the highest adjusted odds-ratio, although the small sample size (only two patients showed this variable) reduced the statistical accuracy.

Degree of instability, activity level and subjective evaluation. The data on pre- and post-operative instability and activity levels are presented in Table IV. Pre-operatively, most patients $(70.5 \%)$ had instability of the shoulder which at least interfered with daily activities. In patients with recurrence of instability, the degree of pre-operative instability was 4 in four patients, 3 in three, and 2 in four. After the operation, seven of these patients had a single recurrence related to a new traumatic event (level 1), whereas of the four remaining patients, one reported instability with sports (level 2), two with activities of daily living (level 3), and one during sleep (level 4). Surgery had significantly decreased the mean degree of instability $(p=0.018)$, in spite of recurrence. Only three patients with instability during activities of daily living or sleep accepted revision.

Sixty patients had participated actively in sports before the onset of shoulder symptoms. Most were recreational athletes. At the final follow-up evaluation, 48 still participated in sports, although the number of patients practising high-risk sports (level 4) had decreased from 37 to 19. Thirteen patients reported that they did not participate in sports because of issues unrelated to their shoulder, such as work or family commitments, or graduation from high school or college. Of the 11 patients with persistent instability, four participated at their previous sporting level, three had decreased the level of participation, and four had stopped all sporting activity. Only two patients reported that they were unable to participate in athletic activities because of instability.

At the latest follow-up, 41 patients $(67.2 \%)$ rated their level of satisfaction as excellent, $12(19.7 \%)$ as good, six $(9.8 \%)$ as fair, and two $(3.3 \%)$ as poor. Five of the eight patients who were dissatisfied had further instability. Two patients developed post-operative stiffness of the shoulder which required mobilisation under anaesthesia in one case, and one patient who had a transient neurapraxia of the musculocutaneous nerve rated the result as fair. Six patients with recurrent post-operative instability rated their result as good or excellent. Only the three patients who needed to be revised recognised that they should have chosen open surgery because of its potentially lower rate of recurrence.

\section{Discussion}

Comparative, prospective and randomised studies have clearly shown that arthroscopic techniques for treatment of instability of the shoulder have higher rates of failure than open surgery, ${ }^{2-4}$ and the rate of recurrence of $18 \%$ observed in our study, although similar to that reported in others, ${ }^{13}$ is not acceptable. However, arthroscopic Bankart repair has clear advantages in comparison with open surgery. ${ }^{1,2}$ These 
objective benefits are supported by the high degree of satisfaction reported by our patients, most of whom recognised that they would choose arthroscopic instead of open stabilisation again, even those with recurrent post-operative instability. The discrepancy between the higher recurrence rates in comparison with open surgery and the objective and subjective benefits of the procedure demand more precise arthroscopic surgery. In addition to technical improvements, it is essential to select patients carefully for this technique. In our study, they were chosen using criteria based on the characteristics of the most frequent type of instability. Other potential sources of problems in the shoulder were excluded, and the patients were operated on using the same surgical technique to obtain a homogeneous group. Thus, the results could be prospectively evaluated to identify key variables associated with an unsatisfactory outcome in order to define precisely the selection criteria for arthroscopic surgery using logistic regression analysis. Furthermore, the definition of recurrent instability was strict and the follow-up long enough to make our conclusions valuable. The results, with more than half the cases of recurrent instability occurring late, confirm the importance of long-term follow-up, and increase the consistency of the technique since recurrences cannot be attributed to the learning curve of the technique.

With regard to the criteria for selection of patients, the first important finding is the effect of the lesion of the anterior glenoid rim on the probability of recurrent instability. This problem has not been approached when studying the arthroscopic treatment of instability in spite of its high prevalence and its deleterious effect on the outcome of surgery. ${ }^{17,19,22,28-30}$ In one study, ${ }^{30}$ although a description of the precise indications for bone grafting in anterior glenoid defects was lacking, only those patients with an osseous defect involving less than $30 \%$ of the glenoid surface were offered arthroscopic stabilisation. Because Itoi et $\mathrm{al}^{22}$ had shown that anterior stability decreases as the size of the glenoid defect increases, those shoulders with fracture of the glenoid rim were divided into two groups in an attempt to define more accurately suitable candidates for arthroscopic Bankart repair. The results confirm that the presence of a fracture of the anterior glenoid rim involving more than $15 \%$ of the glenoid surface represents a contraindication to arthroscopic stabilisation, whereas small bony lesions do not seem to influence the outcome. Therefore, it is advisable to carry out appropriate pre-operative imaging studies to rule out extensive fractures of the glenoid rim.

The second important finding of our study was the independent effect of post-operative sporting activity on outcome. Shoulders which were subjected to contact or overhead sports showed a significantly higher probability of recurrence, and significant trauma involving a contact or overhead activity with the shoulder in abduction and external rotation was noted in all failed cases. It is interesting to note that one of the advantages of arthroscopic techniques of stabilisation over open surgery is a better post-operative range of movement, especially external rotation, ${ }^{1,2}$ a key point for throwing athletes with high functional demands. ${ }^{9}, 31$ However, Pagnani and Dome ${ }^{32}$ demonstrated in a study performed on American football players that open surgery can restore stability while maintaining a good range of movement. We recommend open stabilisation for patients who wish to practise contact or overhead sports but considering our good subjective results, those athletes for whom it is essential to have a full range of movement in abduction and external rotation could be suitable for arthroscopic surgery so long as they understand that there is a higher risk of recurrence and assuming that they have no other risk factors.

Initial studies failed to find any association between age and post-operative recurrent instability, ${ }^{5}$ but the effect of a patient's age on the results of this procedure has been identified in subsequent reports, ${ }^{7,8}$ although a specific age under which open stabilisation should probably not be performed cannot be established precisely. The reasons why age could have an effect on the rate of recurrent instability are not understood. Reeves ${ }^{33}$ noted a tendency for the anterior capsule to fail at the glenoid attachment in young patients, and suggested that the difference could lie at the tissue or cellular level. Nevertheless, trauma with the arm in abduction and external rotation, was the usual mechanism of recurrence in our series, and frequent high-risk activity in younger patients plays an important role.

The presence of ligamentous laxity also had an effect on the outcome after arthroscopic stabilisation, although its influence on the outcome was not statistically determined. Significantly worse results in patients with capsular laxity managed arthroscopically have previously been reported, ${ }^{6,12,14,19}$ and some authors recommend open surgery in these patients. ${ }^{4,12}$ However, capsular laxity has been recognised as an important cause of failure in instability treated by open techniques. ${ }^{17,34,35}$ Our findings coincide with those published in the literature, but according to the results of the statistical analysis we consider that capsular laxity is not a formal contraindication to arthroscopic surgery, and should be considered in the context of the remaining characteristics of the patient. Those patients with arthroscopically documented capsular elongation were treated by a capsular shift in our study. Thus, conclusions concerning other methods of managing capsular laxity such as closure of the rotator interval, which can also be performed arthroscopically and may improve the results in selected patients, ${ }^{14}$ cannot be obtained.

Finally, the remaining variables of gender, age at first dislocation, dominance, the aetiology of instability (traumatic vs atraumatic), the number of pre-operative episodes of instability, the presence of a Bankart or a Hill-Sachs lesion and the number of sutures did not influence the clinical results.

The degree of patient satisfaction with the procedure is very high, even in those cases of recurrent post-operative instability. This could be explained by the fact that our cri- 
teria of failure were strict. In addition, most of the patients in whom the procedure was considered to have failed had improved their level of stability in order not to interfere in their activities, and therefore declined an additional operation. Most of the patients in our series were not athletes who played at highly competitive levels, and they preferred altering the level at which they participated in sports in order to accommodate a potentially unstable shoulder. Gill et $\mathrm{al}^{24}$ showed in a long-term study of open Bankart repair for anterior instability that an adequate range of movement, especially external rotation which allows good function during activities of daily living and recreation, is the highest priority for these patients. Adequate movement was considered to be more important than having a stable and painfree shoulder. Thus, another explanation for our good subjective results may be that movement can be satisfactorily preserved with arthroscopic surgery. Finally, the high level of social acceptance of arthroscopy must also be emphasised. Only one of 65 potential candidates for inclusion in this investigation chose to undergo open surgery, and only one of 11 patients with post-operative instability recognised that they should have had an open instead of arthroscopic surgery to decrease the probability of recurrence.

Our study has a number of weaknesses. Although the investigation was prospective, the patients were not randomised to undergo open or arthroscopic surgery, and thus we do not know the influence of the variables assessed in those treated by open surgery. In addition, only patients who had had the multiple-suture technique were included in order to make the series homogeneous. These conclusions are therefore not necessarily applicable to other arthroscopic techniques based on anchor fixation. However, randomised studies have failed to demonstrate superior results of these techniques over transglenoid multiple suture, ${ }^{6,24}$ and our complication rate is similar to that previously reported. Moreover, our study analyses conditions related to the patient, and not to the technique, which could be helpful in the selection of candidates.

No benefits in any form have been received or will be received from a commercial party related directly or indirectly to the subject of this article.

\section{References}

1. Green MR, Christensen KP. Arthroscopic versus open Bankart procedures: a comparison of early morbidity and complications. Arthroscopy 1993;9:371-4.

2. Karlsson J, Magnusson L, Ejerhed L, et al. Comparison of open and arthroscopic stabilization for recurrent shoulder dislocation in patients with a Bankart lesion. Am J Sports Med 2001;29:538-42.

3. Steinbeck J, Jerosch J. Arthroscopic transglenoid stabilization versus open ancho suturing in traumatic anterior instability of the shoulder. Am J Sports Med 1998;26:373-8.

4. Sperberg A, Hamberg P, Karlsson J, Swärd L, Wredmark T. Comparison of an arthroscopic and an open procedure for posttraumatic instability of the shoulder: a prospective randomized multicenter study. J Shoulder Elbow Surg 2001;10:105-8.

5. Grana WA, Buckley PD, Yates CK. Arthroscopic Bankart suture repair. Am J Sports Med 1993;21:348-53.

6. Manta JP, Organ S, NirschI RP, Pettrone F. Arthroscopic transglenoid capsulolabral repair: five-year follow-up. Am J Sports Med 1997;25:614-18.

7. Pagnani MJ, Warren RF, Altcheck DW, Wickiewicz TL, Anderson AF. Arthroscopic shoulder stabilization using transglenoid sutures: a four-year minimum followup. Am J Sports Med 1996:24:459-67.
8. Torchia ME, Caspari RB, Asselmeier MA, Beach WR, Gayari M. Arthroscopic transglenoid multiple suture repair: 2 to 8 year results in 150 shoulders. Arthroscopy 1997; 13:609-19.

9. Savoie FH, Miller CD, Field LD. Arthroscopic reconstruction of traumatic anterior instability of the shoulder: the Caspari technique. Arthroscopy 1997;13:201-9.

10. Hayashida K, Yoneda M, Nakagawa S, Okamura K, Fukushima S. Arthroscopic Bankart suture repair for traumatic anterior shoulder instability: analysis of the causes of a recurrence. Arthroscopy 1998;14:295-301.

11. DeBerardino TM, Arciero RA, Taylor DC, Uhorchack JM. Prospective evaluation of arthroscopic stabilization of acute, initial anterior shoulder dislocations in young athletes: two- to five-year follow-up. Am J Sports Med 2001;29:586-92.

12. Cole BJ, L'Insalata J, Irrgang J, Warner JJP. Comparison of arthroscopic and open anterior shoulder stabilization: a two- to five-year follow-up study. J Bone Joint Surg [Am] 2000;82-A:1108-14.

13. Kandziora F, Jäger A, Bischof F, et al. Arthroscopic labrum refixation for post-traumatic anterior shoulder instability: suture versus transglenoid fixation technique. Arthroscopy 2000;16:359-66.

14. Gartsman GM, Roddey TS, Hammerman SM. Arthroscopic treatment of anteriorinferior glenohumeral instability: two- to five-year follow-up. J Bone Joint Surg [Am] 2000;82-A:991-1003.

15. Caspari RB, Savoie FH III. Arthroscopic reconstruction of the shoulder: the Bankart repair (suture technique). In: McGinty JB, Caspari RB, Jackson RW, Phoeling GG, eds Operative arthroscopy. Philadelphia: Lippincott-Raven, 1996:695-707.

16. Rowe CR, Patel D, Southmayd WW. The Bankart procedure: a long-term endresult study. J Bone Joint Surg [Am] 1978;60-A:1-16.

17. Rowe CR, Zarins B, Ciullo J. Recurrent anterior dislocation of the shoulder after surgical repair: apparent causes of failure and treatment. J Bone Joint Surg [Am] 1984;66-A:159-68

18. Hovelius L, Augustini GBG, Fredin $\mathbf{O H}$, et al. Primary anterior dislocation of the shoulder in young patients: a ten-year prospective study. J Bone Joint Surg [Am] 1996:78-A:1677-84.

19. Walch G, Boileau P, Levigne $\mathbf{C}$, et al. Arthroscopic stabilization for recurrent anterior shoulder dislocation: results of 59 cases. Arthroscopy 1995;11:173-9.

20. Marcacci M, Zaffagnini S, Petitto A, et al. Arthroscopic management of recurrent anterior dislocation of the shoulder: analysis of technical modifications of the Caspari procedure. Arthroscopy 1996;12:144-9

21. Dora C, Gerber C. Shoulder function after arthroscopic anterior stabilization of the glenohumeral joint using an absorbable tack. J Shoulder Elbow Surg 2000;9:294-8.

22. Itoi E, Lee SB, Berglund LJ, Berge LL, An KN. The effect of a glenoid defect on anteroinferior stability of the shoulder after Bankart repair: a cadaveric study. J Bone Joint Surg [Am]2000;82-A:35-46.

23. Hattrup SJ, Cofield RH, Weaver AL. Anterior shoulder reconstruction: prognostic variables. J Shoulder Elbow Surg 2001;10:508-13.

24. Gill TJ, Micheli LJ, Gebhard F, Binder C. Bankart repair for anterior instability of the shoulder: long-term outcome. J Bone Joint Surg [Am] 1997;79-A:850-7.

25. Kim SH, Ha KI, Kim YM. Arthroscopic revision Bankart repair: a prospective outcome study. Arthroscopy 2002;18:469-82.

26. Wirth MA, Blatter G, Rockwood CA Jr. The capsular imbrication procedure for recurrent anterior instability of the shoulder. J Bone Joint Surg [Am] 1996;78-A: 246-59.

27. Marshall JL, Johanson N, Wickiewicz TL, et al. Joint looseness: a function of the person and the joint. Med Sci Sports Exerc 1980;12:189-94

28. Tamai K, Higashi A, Tomohiro T, Hamada J. Recurrences after the open Bankart repair: a potential risk with use of suture anchors. J Shoulder Elbow Surg 1999:8: $37-41$

29. Kim SH, Ha KI, Cho YB, Ryu BD, Oh I. Arthroscopic anterior stabilization of the shoulder: two to six-year follow-up. J Bone Joint Surg [Am] 2003;85-A:1511-18.

30. Burkhart SS, DeBeer JF. Traumatic glenohumeral bone defects and their relationship to failure of arthroscopic Bankart repairs: significance of the inverted-pear glenoid and the humeral engaging Hill-Sachs lesion. Arthroscopy 2000;16:677-94.

31. Bacilla P, Field LD, Savoie FH. Arthroscopic Bankart repair in a high demand patient population. Arthroscopy 1997;13:51-60.

32. Pagnani MJ, Dome DC. Surgical treatment of traumatic anterior shoulder instability in American football players. J Bone Joint Surg [Am]2002;84-A:711-15.

33. Reeves B. Experiments on the tensile strength of the anterior capsular structures of the shoulder in man. J Bone Joint Surg [Br] 1968;50-B:858-65

34. Young DC, Rockwood CA. Complications of a failed Bristol procedure and their management. J Bone Joint Surg [Am] 1991;73-A:969-81.

35. Levine WN, Arroyo JS, Pollock RG, Flatow EL, Bigliani LU. Open revision stabilization surgery for recurrent anterior glenohumeral instability. Am J Sports Med $2000 \cdot 28 \cdot 156-60$ 